\title{
How does moderate drought affect quantum yield and the regulation of sugar metabolism at low temperature in durum wheat (Triticum durum L.)?
}

\author{
R. KHALIL ${ }^{*, \dagger}$, J. TAJTI ${ }^{* *, \dagger}$, K.Á. HAMOW*** ${ }^{* *}$, K.O. GONDOR ${ }^{* *}$, E. DARKO ${ }^{* *}$, N. ELSAYED ${ }^{*}$, \\ Z. NAGY", G. SZALAI**, T. JANDA ${ }^{* *}$, and I. MAJLÁTH**,+ \\ Botany Department, Faculty of Science, Benha University, 13518 Benha, Egypt* \\ Department of Plant Physiology, Centre for Agricultural Research, ELKH, 2462 Martonvásár, Hungary ${ }^{* *}$ \\ Plant Protection Institute, Centre for Agricultural Research, ELKH, 1022 Budapest, Hungary ${ }^{* * *}$ \\ Cereal Research Non-Profit Ltd., 6724 Szeged, Hungary
}

\begin{abstract}
Currently, limited knowledge is available about the combined effects of drought and light in case of cold stress. The investigation of quantum yield kinetics may help understand how plants utilize light under adverse environmental conditions. In the present study, mild drought helped preserve quantum yield in durum wheat (Triticum durum L.). The time necessary to reach the steady-state actual quantum yield corresponded with the cold sensitivity. Possible underlying mechanisms induced by drought, such as the reduction of malondialdehyde content, accumulation of D-sorbit, and glycine betaine, may have protected photosynthetic apparatus and integrity of light reactions. Low sucrose content at normal growth light might have caused stomatal closing and promoted photorespiration. In contrast to growth light, low light and moderate drought positively affected the sucrose accumulation and maintained glycolysis and energy production at low temperature. Present results emphasized the role of moderate drought as a sufficient acclimation factor against cold in durum wheat.
\end{abstract}

Keywords: cold acclimation; durum wheat; moderate drought; photoprotection; sugar metabolism; tricarboxylic acid cycle.

\section{Introduction}

For terrestrial plants, low temperature and drought are two of the most limiting environmental stresses, exerting overlapping effects in stress acclimation and defence. They trigger secondary metabolic stress, which may induce potential acclimation pathways. This process greatly depends on the severity of the stress and switches the metabolism

\section{Highlights}

- Mild water loss maintained $\mathrm{Y}_{(\mathrm{II})}$ and enhanced fluorescence quenching of wheat in the cold

- Mild drought reduced MDA and elevated glycine betaine to protect light reactions

- Low light facilitated sucrose-glucose conversion under drought
Received 17 February 2021

Accepted 10 May 2021

Published online

${ }^{+}$Corresponding author

phone: +3622569503

e-mail: majlath.imre@atk.hu

Abbreviations: Car - carotenoids; Chl - chlorophyll; DM - dry mass; FI - chlorophyll a fluorescence induction analysis; FM - fresh mass; G1, G2 - groups of abiotic treatment combinations; GB - glycine betaine; LL - low light; MDA - malondialdehyde; $P_{d}-$ point of divergence of the quantum yield curves; ROS - reactive oxygen species; RWC - relative water content; TCA - tricarboxylic acid cycle; $\mathrm{Y}_{(\mathrm{II})}$ - quantum yield of photochemical energy conversion in PSII; $\mathrm{Y}_{(\mathrm{NO})}$ - quantum yield of nonregulated nonphotochemical energy loss in PSII; $\mathrm{Y}_{(\mathrm{NPQ})}$ - quantum yield of regulated nonphotochemical energy loss in PSII.

Acknowledgements: The authors thank G. Vida (Centre for Agricultural Research, Hungary) for providing the cultivars and B. Harasztos for revising the manuscript linguistically. This work was funded by the Hungarian National Research, Development and Innovation Office (K120028).

${ }^{\dagger} \mathrm{R}$. Khalil and J. Tajti contributed equally to this work.

Conflict of interest: The authors declare that they have no conflict of interest. 
to an alarm state (Savvides et al. 2016). The research of combined stress effects is an emerging area of plant biology (Mittler 2006, Pandey et al. 2015, 2017).

Drought and low temperature cause various systemic acclimation responses in plants, such as osmotic adjustment or oxidative burst (Beck et al. 2007, Ruelland et al. 2009, Miura and Furumoto 2013). Plants have evolved common and different tolerance mechanisms and responses regarding chilling and drought stresses (Hussain et al. 2018). The drought itself considerably causes oxidative stress in cells. The roles of enzymatic and nonenzymatic antioxidants were reviewed by Ahmad et al. (2010). Water deficiency enhances the activities of antioxidant enzymes superoxide dismutase, guaiacol peroxidase, catalase, ascorbate peroxidase and gently alters glutathione, ascorbic acid, $\alpha$-tocopherol, and $\mathrm{H}_{2} \mathrm{O}_{2}$ contents. More than sixty $\mathrm{H}_{2} \mathrm{O}_{2}$-responsive transcription factors involved in abiotic stresses such as cold or drought were identified. Drought induces $\mathrm{Ca}^{2+}$-dependent signalling which activates LEA-type genes. LEA-like proteins may function as chaperones. Further, soluble sugars, including sucrose, and sugar alcohols are sequestered in vacuoles in response to oxidative stress and protect membranes against freezing and drought stress by scavenging hydroxyl radicals. On the other hand, reactive radicals have an important role in the signalling of stress defence (Kohli et al. 2019).

Light is pivotal for carbon assimilation, however, under adverse growth conditions, the output of the light reactions related to photosynthesis is a major source of free radicals which has several undesirable effects in the plant cell (Sharma et al. 2012). This Janus-faced nature of light reactions is well-demonstrated when leaves are exposed to ambient light under unfavourable environmental conditions, which often results in oxidative stress on the thylakoid membranes due to the imbalance between light capture and utilization (Janda et al. 2014). The formation of oxidative agents disrupts the membranes, including thylakoid membranes (Keren and Krieger-Liszkay 2011, Nejadsadeghi et al. 2015). Differences in light intensity also lead to changes in photosynthetic pigment composition through the redox potential generated during photosynthesis (Pizarro and Stange 2009). When drought limits $\mathrm{CO}_{2}$ availability, it strongly contributes to the formation of ROS. Regarding the reparation rate of proteins, oxidative stress inhibits translation elongation factors. In an in vitro study of cyanobacterial translation, hydrogen peroxide oxidized elongation factor $G$, which resulted in the inactivation of translation (Takahashi and Murata 2008).

Damages of the thylakoid membrane and photosystems modify the ultrastructure of the photosynthetic machinery, thus affect the utilization of the absorbed quanta (Kramer et al. 2004, Chan et al. 2012). The energy of the absorbed quantum is utilised in several ways, which can be measured as follows: the quantum yield of photochemical energy conversion in PSII [ $\left.\mathrm{Y}_{(\mathrm{II})}\right]$, the quantum yield of regulated nonphotochemical energy loss in PSII [ $\mathrm{Y}_{(\mathrm{NPQ})}$ ], and quantum yield of nonregulated nonphotochemical energy loss in PSII [Y $\left.\mathrm{Y}_{(\mathrm{NO})}\right]$ (Klughammer and Schreiber 2008). Detection of chlorophyll (Chl) fluorescence is a widely used tool for determining these parameters and evaluating the light-utilization capacity of plants (Kalaji et al. 2016, Nath et al. 2017).

The regulation of harvest and utilization of light is flexible, allowing the output of energy-storing reactions to be regulated and ensuring dynamic responses to the changing environment (Kramer et al. 2004). Photosynthetic electron transport was found to remain undisturbed under mild water stress (Cornic and Fresneau 2002) and oppositely, actual quantum yield decreased upon the increasing level of water shortage (Cornic and Briantais 1991). During stress conditions, several scavenging mechanisms have evolved to decrease the loss in $\mathrm{Y}_{(\mathrm{II})}$ caused by low temperature and drought. One of the major mechanisms, an osmotic adjustment has great importance as a systemic stress response during simultaneous cold and drought stresses (Saxena et al. 2013). Glycine betaine (GB) is an amphoteric quaternary ammonium compound with versatile roles, such as protecting cells through osmotic adjustment, stabilizing proteins, and scavenging reactive oxygen species (ROS). GB also has a role in the defence of the photosynthetic apparatus via maintaining the integrity of the ultrastructure of chloroplasts and mitochondria (Mäkelä et al. 2000). GB is formed as the result of twostep oxidation of choline via betaine aldehyde. In higher plants, the final reaction is catalysed by betaine aldehyde dehydrogenases, which are localized in the stroma (Ahmad et al. 2013).

Metabolites, including metabolisable sugars, are the final products of the process of cell control, which are not only closely related to plant growth and development but also the effect of different environmental factors (Fiehn 2002, Zhang et al. 2016). From a metabolic point of view, it is possible to profoundly understand the relationship between plants and the environment. Osmotic stress is imposed by drought, cold, and low light stress, which can lead to loss of turgor. The osmotic regulators, including amino acids, organic acids, and soluble sugars, are primarily small molecular organic compounds (Evers et al. 2010). Some major small molecular metabolites, such as sucrose, fructose, glucose, raffinose, lactic acid, pyruvic acid, and GB, are known to accumulate under drought stress in large amounts, which can decrease the capacity of cytoplasmic water and preserve environmental stability (Chemikosova et al. 2006, Amin et al. 2009). A variety of metabolic pathways, including glycolysis, tricarboxylic acid cycle (citric acid cycle, TCA), sugar biosynthesis, and amino acid biosynthesis, also include plant's reaction to drought stress (Guo et al. 2018).

Considering global climate change, enhancing crop productivity is a crucial question in agronomy. Priming (chemical hardening) is a possible way to reach better crop productivity. Numerous experimental and applied methods are known and studies are available in the literature when crops were tested with several chemical compounds for priming purposes against combined stresses. Sulfur enrichment in soil successfully enhanced the combined drought and phosphorus-deficiency stress tolerance in maize (Kaya et al. 2020). Exogenous 24-epibrassinolide induced nitric oxide generation and nitric oxide-mediated 
antioxidant defence systems against drought in pepper plants (Kaya et al. 2019). Alpha-tocopherol treatment improved yield parameters in bread wheat under drought (Ali et al. 2019), while exogenous salicylic acid and hydrogen peroxide mitigated drought stress in rice (Sohag et al. 2020).

However, besides priming experiments, acclimation procedures against combined abiotic stress factors, which are aimed to improve crop fitness and yield, are less well-known. The simultaneous occurrence of certain abiotic stresses results in plant stress defence responses that cannot be directly extrapolated from the separate responses of plants to each of the different stresses (Mittler 2006). Plant responses to a combination of chilling and drought stresses are unique from those to individual stress and adversely affect plant growth due to the complexity of physiological and biochemical disruptions. In general, the simultaneous cold and drought effect is even worse for plant life compared to individual stress acts (Hussain et al. 2018). Like other combinations of abiotic stress factors, the physiology of simultaneous cold, drought, and illumination has not been investigated in more detail (Savvides et al. 2016). Worth mentioning is that the effect on the individual greatly depends on the severity of the stress factors.

Moderate drought was proven as a sufficient acclimation factor influencing nitrate reduction and steady-state $\mathrm{Y}_{(\mathrm{II})}$ at low temperatures in durum wheat (Majláth et al. 2016). A recent observation also confirmed that moderate drought stress stabilized the primary quinone and secondary quinone acceptors in PSII (Leverne and Krieger-Liszkay 2021). Besides the knowledge of the steady-state level of actual quantum yield, the question is still open how moderate drought alters the kinetics of $Y_{(I)}$ related to the cold tolerance, and how photosynthetic sugar metabolism is affected by regulated water deficiency in the cold.

In the present study, a detailed analysis of chlorophyll fluorescence induction kinetics was carried out in order to dissect the effects of the combination of light and moderate drought and low-temperature stress on $Y_{(I I)}$ in durum wheat and to reveal the hypothesized photoprotective processes. The main goal of the study was to clarify how regulated water stress can maintain metabolism under low-temperature conditions. The relationship was studied between the changes in $\mathrm{Y}_{(\mathrm{II})}$, the level of lipid peroxidation, photosynthetic pigment composition, GB, and different sugars and organic acids, with special regard to their dependence on light. Changes in certain sugars may explain the light-utilization capability improved by mild drought during suboptimum temperature conditions. The relationship of the kinetic changes in $Y_{\text {(II) }}$ to the cold tolerance of cultivars was also unraveled.

\section{Materials and methods}

Plant growth: Triticum durum L. plants [cv. Mv Makaróni (cold-tolerant) and cv. MvTD10-10 (cold-sensitive)] were grown according to the experimental procedure described by Majláth et al. (2016) with small modifications. The cultivars were selected based on their importance as new breeding products with unexplored physiology and preliminary freezing survival tests carried out in growth chambers. The plants were grown at $21^{\circ} \mathrm{C}$ with a PPFD of $250 \mu \mathrm{mol} \mathrm{m}^{-2} \mathrm{~s}^{-1}$ (growth light).

From the $10^{\text {th }}$ day, half of the plants were grown under normal irrigation $(35 \pm 5 \%$ relative soil humidity, CONTROL), while another half was exposed to moderate drought $(15 \pm 5 \%$ relative soil humidity, CONTROL+DROUGHT) for drought-preconditioning purpose.

From the $21^{\text {st }}$ day (3-week-old plants), plants were divided into two populations: (1) CONTROL and CONTROL+DROUGHT plants were further grown at $21^{\circ} \mathrm{C}$, and (2) $5^{\circ} \mathrm{C}$ suboptimum temperature for 2 weeks was used for a cold-hardening purpose. The populations growing in the cold (COLD, COLD+DROUGHT) were illuminated with normal light and half of them with reduced, low light $\mathrm{PPFD}=50 \mu \mathrm{mol} \mathrm{m} \mathrm{m}^{-2} \mathrm{~s}^{-1}[$ low light (LL)] (COLD+LL, COLD+DROUGHT+LL) in order to investigate the impact of photoinhibition under normal grow light and LL conditions in the cold. The experimental setup is illustrated for an easier overview in Fig. 1S, supplement.

Soil humidity was inspected daily using a soil moisture meter with an SM200 sensor (Delta-T Devices, Cambridge, UK). The third fully developed leaves of the 5-week-old plants were harvested for the physiological and biochemical assays.

Chlorophyll $a$ fluorescence induction (FI): The FI analysis was carried out using pulse amplitude modulated fluorometer (PAM) with a blue LED-Array Illumination Unit IMAG-MAX/L $(\lambda=450 \mathrm{~nm})$ (Imaging-PAM MSeries, Walz, Effeltrich, Germany). After a 30-min dark adaptation of the third fully developed leaves, quenching analysis was carried out at laboratory temperature (adapted state) using a continuous $270 \mu \mathrm{mol}$ (photon) $\mathrm{m}^{-2} \mathrm{~s}^{-1}$ actinic light intensity until the steady-state level of photosynthesis was reached (duration: $15 \mathrm{~min}$ ). During the quenching period, with 0.8-s duration, saturation light intensity of 3,000 $\mu \mathrm{mol}$ (photon) $\mathrm{m}^{-2} \mathrm{~s}^{-1}$ with $30-\mathrm{s}$ frequency was applied provided by the $I M A G-M A X / L$ unit. The major quantum yield parameters $\left[\mathrm{Y}_{(\mathrm{II})}, \mathrm{Y}_{(\mathrm{NPQ})}, \mathrm{Y}_{(\mathrm{NO})}\right]$ were calculated during the entire analysis as described by Klughammer and Schreiber (2008).

Relative water content (RWC): Dehydration caused by drought stress greatly alters the water status of leaf tissues. To take into consideration the effect of water loss on the analysed tissue mass, the metabolite levels determined in DROUGHT-treated samples were corrected by RWC as it was described by Majláth et al. (2016).

Photosynthetic pigments: Total chlorophyll $(\mathrm{Chl})$ and carotenoid (Car) contents were determined using the adaptation of the method described by Lichtenthaler (1987). The central part of the third fully developed leaf $(50 \mathrm{mg})$ was ground in $4 \mathrm{ml}$ of ice-cold $80 \%$ acetone $($ Sigma $)$. Crude extracts were centrifuged at $12,000 \times g$ for $10 \mathrm{~min}$ and kept at $0^{\circ} \mathrm{C}$ in the dark before the measurement. 
Pigment contents were measured spectrophotometrically at 750, 664, 646, and $470 \mathrm{~nm}$ (Shimadzu UV-VIS 160A, Kyoto, Japan) and calculated as follows: Chl $a=$ $12.24 \times \mathrm{A}_{664}-2.79 \mathrm{~A}_{646}$, Chl $b=21.5 \times \mathrm{A}_{646}-5.1 \times \mathrm{A}_{664}$, Chl $(a+b)=7.15 \times \mathrm{A}_{664}+18.71 \times \mathrm{A}_{646}$, and xanthophylls + $\mathrm{Car}=\left(1,000 \times \mathrm{A}_{470}-1.82 \times \mathrm{Chl} a-85.02 \times \mathrm{Chl} b\right) / 198$, and expressed in $\mathrm{mg}$ (pigment) $\mathrm{g}^{-1}(\mathrm{DM})$.

Lipid peroxidation was estimated based on the measurement of malondialdehyde (MDA) content. Leaf tissue of $0.3 \mathrm{~g}$ of was ground in $600 \mu \mathrm{l}$ of $0.1 \%(\mathrm{w} / \mathrm{v})$ trichloroacetic acid. Afterward, extracts were centrifuged at $12,000 \times \mathrm{g}$ for $10 \mathrm{~min}$. The supernatant of $300 \mu \mathrm{l}$ was mixed with $2 \mathrm{ml}$ of $0.5 \%(\mathrm{w} / \mathrm{v})$ thiobarbituric acid in $20 \%(\mathrm{w} / \mathrm{v})$ trichloroacetic acid and incubated at $90^{\circ} \mathrm{C}$ for $30 \mathrm{~min}$.

The MDA equivalent component content was measured spectrophotometrically (Shimadzu UV-VIS 160A, Kyoto, Japan) at $532 \mathrm{~nm}$, then the nonspecific absorption at $600 \mathrm{~nm}$ was subtracted and expressed in $\mathrm{nM}(\mathrm{MDA}) \mathrm{g}^{-1}(\mathrm{DM})$ (Thomas et al. 2004).

GB concentration was measured using the modified method of Grieve and Grattan (1983). Leaf tissue of $0.5 \mathrm{~g}$ was ground in $6 \mathrm{ml}$ of ice-cold ultrapure water (MQ). Crude extracts were centrifuged at $12,000 \times g$ for $10 \mathrm{~min}$ at $0^{\circ} \mathrm{C}$. Thereafter, $0.7 \mathrm{ml}$ of supernatant was combined with an equivalent amount of ice-cold $2 \mathrm{~N} \mathrm{H}_{2} \mathrm{SO}_{4}$ and incubated on ice for $1 \mathrm{~h}$. Afterward, $0.6 \mathrm{ml}$ of ice-cold Lugol solution ( $20 \mathrm{~g}$ potassium iodide with $15.7 \mathrm{~g}$ iodine in $100 \mathrm{ml}$ of water) was added. Reaction mixtures were incubated on ice in the dark for $24 \mathrm{~h}$. Samples were centrifuged at $12,000 \times \mathrm{g}$ for $10 \mathrm{~min}$ at $0^{\circ} \mathrm{C}$. The supernatant was gently removed by pipetting, then the pellet was diluted in $4.5 \mathrm{ml}$ of 1.2-dichloroethane with vigorous shaking at room temperature.

The GB-equivalent betaine-periodate crystal content was measured spectrophotometrically (Shimadzu UV-VIS $160 \mathrm{~A}$, Kyoto, Japan) at $365 \mathrm{~nm}$ and expressed in $\mathrm{mg}(\mathrm{GB})$ $\mathrm{g}^{-1}(\mathrm{DM})$. Dilutions of $1 \mathrm{mg}$ (betaine) $\mathrm{ml}^{-1}$ (Sigma) to 50 , $100,200,300$, and $400 \mu \mathrm{g} \mathrm{ml}^{-1}$ (in $1 \mathrm{~N} \mathrm{H}_{2} \mathrm{SO}_{4}$ ) solution were used as standard.

Untargeted polar metabolite identification and quantitation by GC-MS: For the measurement of polar metabolites (organic acids, sugars, and sugar alcohols), $200 \mathrm{mg}$ of the fresh mass of plant material was used for extraction and measurements exactly as described by Janda et al. (2021). Briefly, plant material was extracted by methanol:water, then liquid-liquid partitioned with chloroform (for cleanup of apolar compounds), and part of the methanol:water supernatant was evaporated in a vacuum centrifuge, and methoxyaminated with methoxyamine hydrochloride in pyridine, followed by derivatization (silylation) with N-methyl-N-(trimethylsilyl)-trifluoroacetamide (MSTFA). Samples were injected in split mode into a GC-MS-QP2010 (Shimadzu, Kyoto, Japan) and separation was achieved using a $30 \mathrm{~m} \times 0.25 \mathrm{~mm} \times$ $0.25 \mu \mathrm{m} \mathrm{J \& W}$ HP5ms UI capillary column (Agilent Technologies, USA), where helium was used as a carrier gas with a constant flow mode. Mass spectrometric detection was done in a scan mode with $10 \mathrm{~Hz}$. Data analysis was carried out using Shimadzu GC-MS Solution Postrun analysis software searching the Wiley $9^{\text {th }}$ edition mass spectral database and utilizing retention indices (RI). For additional identification and confirmation, the NIST17 Mass spectral \& RI database was also searched through the use of the AMDIS and MS Search v. 2.3 software. Identified and quantified compounds were summarized in Table 1S (supplement) including their respective retention time, n-alkane based Kovats-retention index and their respective EIC quantitative ion or indication of TIC quantitation and availability of reference material.

Statistical analyses: Means and standard deviations were calculated from at least five biological replicates. Boxplot illustration, one-way analysis of variance (ANOVA) combined with the Tukey's post hoc test $(p \leq 0.05)$ and PCA were carried out using the Agricolae, FactoMineR, factoextra, and ggplot 2 packages in $R$ programming environment (v. 4.0.3).

\section{Results}

Plant growth under different watering and light status: Mv Makaróni and MvTD1-10 durum wheat plants showed different appearances under the combined irrigation and illumination treatments when applied during the cold period. The growth of plants under COLD, COLD+LL, COLD+DROUGHT, COLD+DROUGHT+LL is shown in Fig. 2S (supplement). It can be seen that MvTD10-10 individuals may keep their turgor pressure in a better manner, and lesser effect of wilting could be observed visually under COLD+DROUGHT and COLD+DROUGHT+LL, as compared to $\mathrm{Mv}$ Makaróni (Fig. 2SB). The state of wheat plants shows that water deprivation may not be deleterious and possibly induces defence response at physiological and biochemical levels.

Moderate drought helps to retain $Y_{(\text {II) }}$ at low temperature: Besides the steady-state quantum yield values, focusing on the initial characteristics of kinetic curves provides insight into how the cold sensitivity of genotypes and various combinations affected the light-utilization capability. To understand how moderate water loss influences quantum yield under the combinations of various abiotic factors, two groups $(\mathrm{G})$ were established from six treatment combinations as shown in Fig. 1. G1 includes DROUGHT, COLD, COLD+LL, and G2 are CONTROL, COLD+DROUGHT, COLD+DROUGHT+LL, based on the current hypothesis that plants under COLD and COLD + LL can be expectedly more injured by low temperature than under COLD+DROUGHT and COLD+ DROUGHT+LL. Additionally, CONTROL was attached to G2 and DROUGHT to G1, in order to clarify how quantum yield values resemble or differ from the values detected at the growth temperature. Different light intensities were preset to understand better how the level of excitation pressure of PSII interacts with the effect of mild drought preconditioning. Boxplot analysis was carried out to compare the distribution of quantum yield 


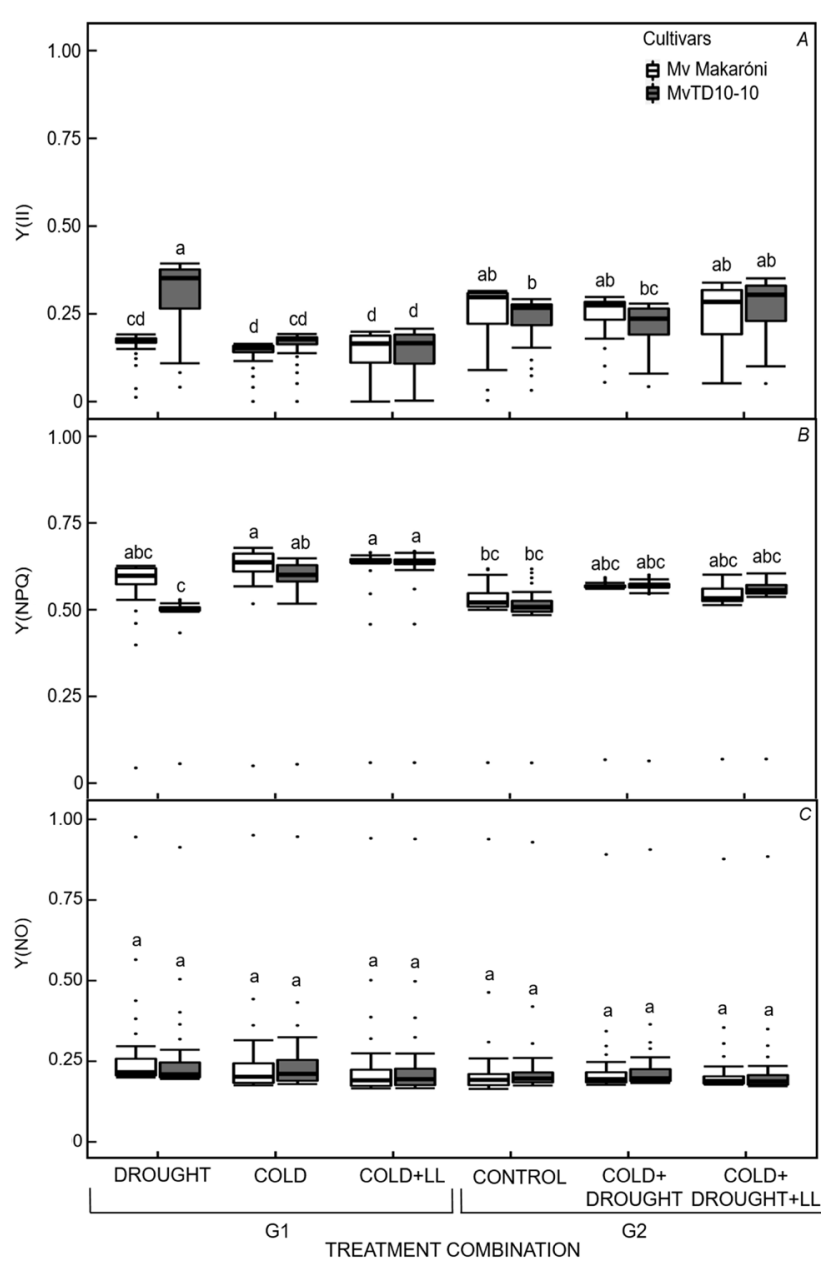

Fig. 1. The boxplot representation of the changes in the $(A)$ actual quantum yield $\left[\mathrm{Y}_{(\mathrm{II})}\right],(B)$ quantum yield of regulated energy dissipation $\left[\mathrm{Y}_{(\mathrm{NPQ})}\right]$, and $(C)$ quantum yield of nonregulated energy dissipation $\left[\mathrm{Y}_{(\mathrm{NO})}\right]$ parameters derived from Chl $a$ fluorescence quenching kinetics in the cold-tolerant Mv Makaróni and cold-sensitive MvTD10-10 durum wheat cultivars under all experimental treatment groups (CONTROL, DROUGHT, COLD, COLD+DROUGHT, COLD+LL, COLD+DROUGHT+ LL). Two boxes belong to every treatment group: Mv Makaróni (left box) and MvTD10-10 (right box). Each box includes the full quantum yield kinetic data of a treatment group. The 25 and $75 \%$ quartiles are drawn using a box, the median is shown with a horizontal line inside the box and outliers are shown as small circles. Different letters indicate significant differences between the treatments at $p \leq 0.05$ using Tukey's post hoc test.

kinetic data, the magnitude of changes (- values mean a decrease and + values mean a percentage of increase) between COLD vs. DROUGHT and COLD+LL vs. COLD+DROUGHT+LL. Thereafter significance levels of the above-mentioned parameters between treatment groups were investigated.

The boxplot comparison of quantum yield parameters including $\mathrm{Y}_{\text {(II) }}$ demonstrated that actual quantum yield values recorded during the entire quenching period were close to each other both in G1 and G2 groups. Both median values and interquartile ranges were mostly in agreement with each other. The asymmetric data distribution within boxes derives from the characteristics of the kinetic curves, namely that a greater number of repetitions of the kinetic data was recorded during the steady-state phase of the quenching period. Steady-state data are more similar to each other than to the monotonically changing initial data.

Treatments of G1 showed lower $Y_{(I I)}$ values as compared to $\mathrm{G} 2$ (Fig. 1A). Significant increases in $\mathrm{Y}_{(\mathrm{II})}$ were found between COLD and COLD+DROUGHT in Mv Makaróni (+40\%) and MvTD10-10 (+24\%). Moderate drought caused +38 and $+41 \%$ changes in the cultivars under COLD+LL and COLD+DROGHT+LL comparison, too. In contrast to $\mathrm{Y}_{(\mathrm{II})}$, values of $\mathrm{Y}_{(\mathrm{NPQ})}$ were lower in $\mathrm{G} 2$ and higher in G1. At grow light, $\mathrm{Y}_{(\mathrm{NPQ})}$ decreased to -11 and $-5 \%$ in Mv Makaróni and MvTD10-10, respectively. Further, there was $-15 \%$ loss found in Mv Makaróni and $-11 \%$ in MvTD10-10 at LL. The values of $\mathrm{Y}_{(\mathrm{NO})}$ were less influenced by the abiotic factors and their combinations. Moderate drought acclimation caused $-7 \%$ loss in $\mathrm{Y}_{\text {(NO) }}$ under the growth and low light in both genotypes (Fig. 1B,C).

When inspecting the kinetic curves (Fig. 3S, supplement), the divergence of G1 and G2 was found to be different on a timescale that was genotype-dependent. In contrast to the cold-tolerant Mv-Makaróni, the $\mathrm{Y}_{\text {(II) }}$ curves G1 and G2 were closer to each other in MvTD10-10. In Mv Makaróni, the G1 and G2 groups were more separated. DROUGHT curve was associated with G1 in Mv Makaróni, whilst in MvTD10-10, it was closer to G2. This denotes a temporal shift in the $\mathrm{P}_{\mathrm{d}}$ values $\left(\mathrm{P}_{\mathrm{d} 1}\right.$ and $\left.\mathrm{P}_{\mathrm{d} 3}\right)$ of the cultivars [Mv Makaróni (5.5 min) and MvTD10-10 (8 min)] $\left(\mathrm{P}_{\mathrm{d}}\right.$, point of divergence of curves, i.e., the time on the $x$-axis indicating the divergence of the curves of quantum yield parameters) (Fig. 3S $A, B$ ). Besides the distinction of $\mathrm{G} 1$ and G2 curves, MvTD10-10 plants seemed to be more cold sensitive under COLD+LL conditions than that of Mv-Makaróni, when the lowest $\mathrm{Y}_{\text {(II) }}$ values were detected during the initial phase of quenching $\left(\mathrm{P}_{\mathrm{d} 2} 2-5.5 \mathrm{~min}\right)$ (Fig. $3 \mathrm{~S} B$ ). The above findings showed that the time required to reach the steady-state level of photosynthesis was different in each acclimations and it may be in agreement with the cold sensitivity of cultivars.

The shape of the $\mathrm{Y}_{(\mathrm{NPQ})}$ curves can be related to the changes observed in $\mathrm{Y}_{(\mathrm{II})}$, with a higher $\mathrm{Y}_{(\mathrm{NPQ})}$ level for $\mathrm{G} 1$ and a lower level for G2 (Fig. 3SC,D). Based on the observed $\mathrm{Y}_{(\mathrm{II})}$ kinetics, the cold-sensitive MvTD10-10 plants exhibited the lowest $\mathrm{Y}_{\text {(II) }}$ values with the highest $\mathrm{Y}_{(\mathrm{NPQ})}$ under $\mathrm{COLD}+\mathrm{LL}$ during the initial phase of quenching $\left(\mathrm{P}_{\mathrm{d} 2}\right.$ and $\left.\mathrm{P}_{\mathrm{d} 4}\right)$ (Fig. 3SB,D) which can show that the cold-sensitive cultivar acclimated to actinic light less effectively.

As it was observed on the boxplot comparison (Fig. 1C), only minor differences were found between G1 and G2 curves of $\mathrm{Y}_{(\mathrm{NO})}(\mathrm{Fig}$. 3SE,F). Light itself had no significant effect on either $\mathrm{Y}_{(\mathrm{II})}$ or $\mathrm{Y}_{(\mathrm{NPQ})}$ or $\mathrm{Y}_{(\mathrm{NO})}$ in the cold. All data of $\mathrm{Y}_{(\mathrm{II})}, \mathrm{Y}_{(\mathrm{NPQ})}$, and $\mathrm{Y}_{(\mathrm{NO})}$ can be found in Table $2 \mathrm{~S}$ (supplement). 
Total chlorophyll (Chl), carotenoid (Car), and MDA content: To reveal the underlying mechanisms of the changes observed in quantum yield parameters, photosynthetic pigment composition and MDA content were determined. At $21^{\circ} \mathrm{C}$, total $\mathrm{Chl}$ content (Chl $a$ and $\mathrm{Chl} b$ ) only decreased in response to moderate drought in MvTD10-10. The cold caused a further decline in the Chl content at both water regimes as compared to the control temperature. Light-dependent changes were only found in the case of normal water supply, the Chl content was significantly higher at low light than that at normal light. In COLD vs. COLD+DROGHT and COLD+LL vs. COLD+DROGHT+LL comparisons, total Chl contents changed with -26 and $-31 \%$ in Mv Makaróni and -66 and $-46 \%$ in MvTD10-10, respectively (Fig. 2A). Similar differences were observed in the Car content as compared to $\mathrm{Chl}$ content under cold and LL conditions ( -30 and $-24 \%$ in $\mathrm{Mv}$ Makaróni and -101 and $-38 \%$ in MvTD10-10) (Fig. 2B).

The content of MDA was investigated in order to see how mild drought pretreatment affects lipid peroxidation. The MDA content remained unchanged at CONTROL and DROUGHT at growth temperatures. However, a significantly higher MDA content was observed at COLD in both cultivars. This cold-induced increase of MDA was diminished by both low light and moderate drought (COLD+DROUGHT, COLD+LL, COLD+DROUGHT+LL states) in both cold-tolerant and sensitive cultivars. The MDA content was reduced by drought acclimation under growth light with -45 and $-31 \%$ in Mv Makaróni and MvTD10-10, respectively. It also decreased under LL by $-4 \%$ in MvTD10-10 and Mv Makaróni, however, MDA slightly increased $(+17 \%)$ by drought (Fig. 3A).

Low light induces a greater degree of GB accumulation than moderate drought: The content of GB was found to respond positively to drought at both growth and low temperatures. Low temperature and normal water supply (COLD state) for two weeks did not alter the GB content. Nevertheless, normal watering and low light (COLD+LL) and mild drought during cold (COLD+DROUGHT, COLD+DROUGHT+LL) significantly increased the foliar GB concentration. GB remarkably increased by drought pretreatment at growth light (+86 and $+91 \%$ in $\mathrm{Mv}$ Makaróni and MvTD10-10, respectively) and LL (+52 and $+63 \%$ in Mv Makaróni and MvTD10-10, respectively) conditions. The accumulation of GB additionally increased by LL irrespective of the water supply (Fig. 3B).

Sugars and organic acids were differentially affected by combined abiotic factors: The content of six sugars, eight organic acids, and sugar alcohol was also determined in order to see how assimilation functioned and, on the other hand, how the osmotic defence was triggered by the controlled water deficiency. PCA biplot representation showed that the metabolic patterns of populations grown at $21{ }^{\circ} \mathrm{C}$ optimum growth temperature (CONTROL and DROUGHT) were entirely different from all populations grown in the cold. They formed separated clusters.

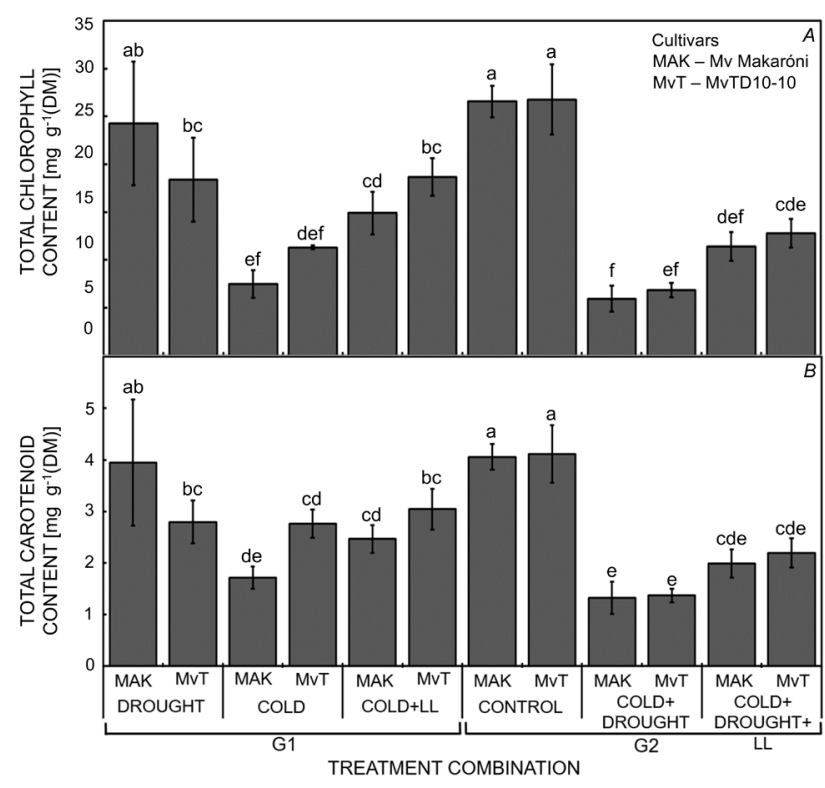

Fig. 2. (A) Total chlorophyll and $(B)$ carotenoid content in the cold-tolerant Mv Makaróni and cold-sensitive MvTD10-10 durum wheat cultivars. Data represent means \pm SD calculated from the data of five plants per treatment. Different letters indicate significant differences between the treatments at $p \leq 0.05$ using Tukey's post hoc test.

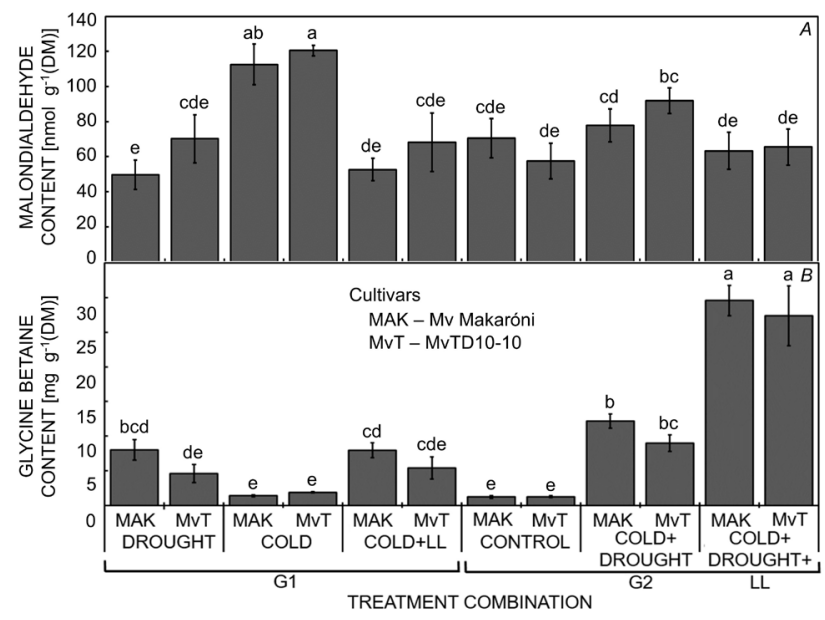

Fig. 3. (A) Changes in malondialdehyde content and (B) glycine betaine content in the cold-tolerant Mv Makaróni and coldsensitive MvTD10-10 durum wheat cultivars. Data represent means \pm SD calculated from the data of five plants per treatment. Different letters indicate significant differences between the treatments at $p \leq 0.05$ using Tukey's post hoc test.

Regarding groups of low temperature, COLD, COLD+ DROUGHT, and COLD+LL, COLD+DROUGHT+LL could also be separated. In Mv Makaróni, changes of D-ribose, pyruvic acid, and succinic acid contents caused different metabolism under low light, whilst sucrose and raffinose were mostly involved in the separation of the 
COLD group. D-turanose was further responsible for the altered metabolism of COLD+DROUGHT plants (Fig. 4SA, supplement). In MvTD10-10, alterations of fructose, glucose, raffinose, pyruvic and succinic acid contents were pronouncedly involved in sugar metabolism under COLD+DROUGHT $(+\mathrm{LL})$. Sucrose was primarily attributed to the COLD group and lactic acid to the COLD+DROUGHT group (Fig. 4SB). Most of the metabolites showed closely similar changes and contents between CONTROL and DROUGHT and all of the
COLD treatment combinations, namely that there were no significant changes found between CONTROL and DROUGHT at growth temperature and cold hardening generally increased metabolite contents (Fig. 4).

Nonmetabolizable sugars, such as D-turanose, significantly increased in Mv Makaróni at COLD (+84\%) and COLD+DROUGHT (+93\%) and in MvTD10-10 (+82 and $+57 \%$, respectively) (Fig. $4 L$ ). Due to low light, the amount of D-turanose dropped to the control level. Raffinose concentration equally rose in the cold independently of
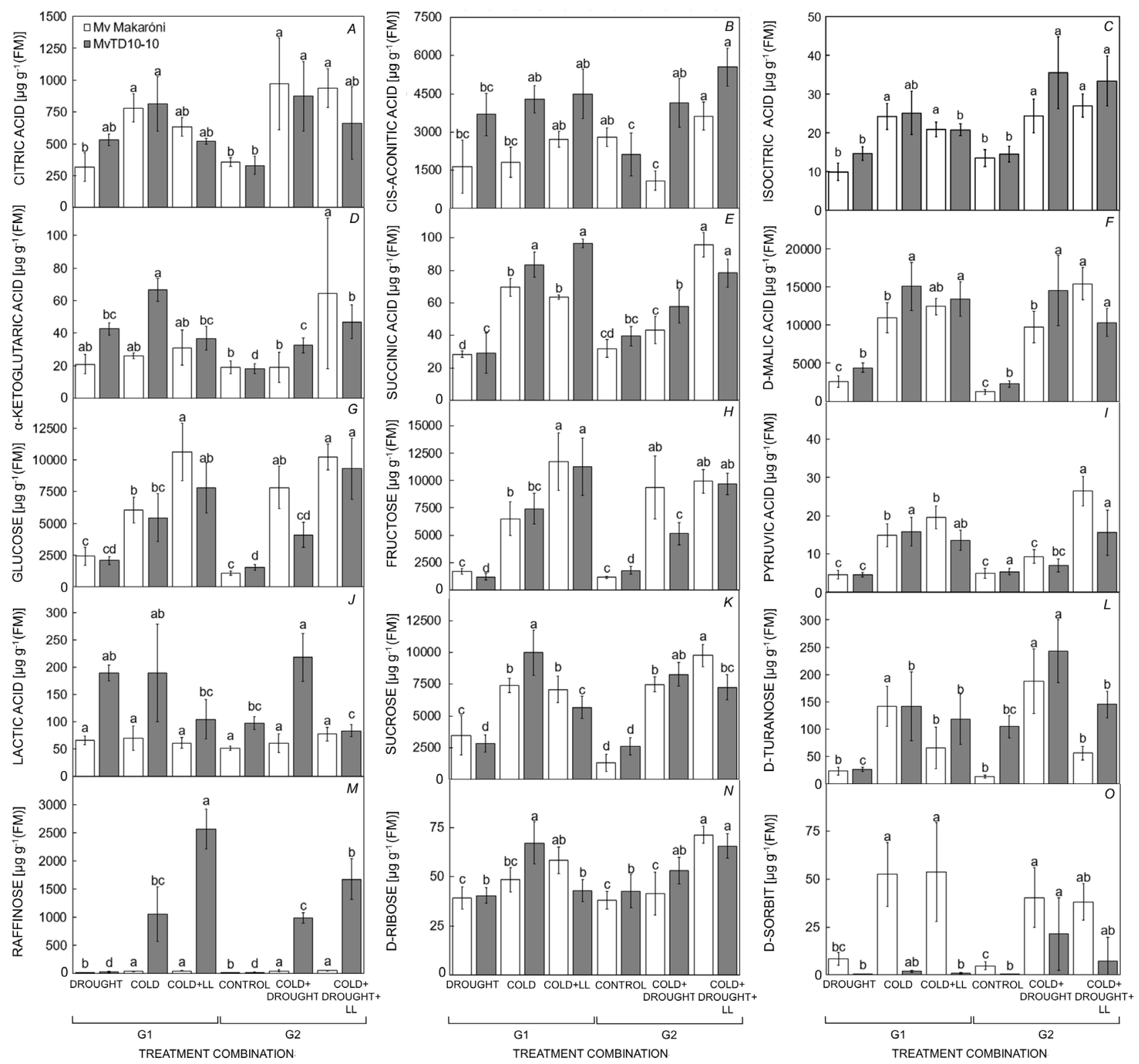

Fig. 4. Citric acid $(A)$, cis-aconitic acid $(B)$, isocitric acid $(C)$, alpha-ketoglutaric acid $(D)$, succinic acid $(E)$, D-malic acid $(F)$, glucose $(G)$, fructose $(H)$, pyruvic acid $(I)$, lactic acid $(J)$, sucrose $(K)$, D-turanose $(L)$, D-ribose $(M)$, raffinose $(\mathrm{N})$, and D-sorbit $(O)$ contents in the leaf of cold-tolerant durum wheat cultivar Mv Makaróni and cold-sensitive cultivar MvTD10-10. Data are mean \pm SD. Different letters indicate significant differences between the six treatment groups in the case of each metabolites per cultivar (CONTROL, DROUGHT, COLD, COLD+DROUGHT, COLD+LL, COLD+DROUGHT+LL) in the case of each compounds at $p \leq 0.05$ using Tukey's post hoc test. 
watering status and light. This increase was around 10-fold in Mv Makaróni and 100- to 200-fold in MvTD10-10 as compared to the CONTROL (Fig. $4 N$ ).

Amongst the sugars of glycolysis and glucose metabolism, D-ribose content was mostly elevated by low light and drought, in Mv Makaróni by $+18 \%$ and in MvTD10-10 by $+34 \%$ (Fig. $4 M$ ). Fructose, glucose, and sucrose increased in the cold (Fig. $4 G, H, K$ ). Moderate drought did not significantly affect their contents. Low light, however, further elevated the fructose and glucose concentrations under DROUGHT conditions (glucose: +23 and $+56 \%$, fructose: +6 and $+47 \%$ in Mv Makaróni and MvTD10-10, respectively). Sucrose contents were slightly elevated under drought and low light in Mv Makaróni $(+23 \%)$ and in MvTD10-10 (+22\%) as compared to normal light conditions (Fig. $4 K$ ).

Pyruvic acid content was slightly influenced by drought at low light $(+26 \%)$ in Mv Makaróni only. COLD $(+67 \%)$, COLD+LL (+75\%), and COLD+DROUGHT+LL (+81\%) treatments increased pyruvic acid content as compared to the CONTROL (Fig. 4I). The lactic acid content was not affected by combined stress treatments in Mv Makaróni and higher lactic acid contents were found in the case of DROUGHT, COLD, and COLD+DROUGHT in MvTD10-10 (Fig. 4J).

The quantities of organic acid members of the citric acid cycle were found to be significantly altered by the dependence on light (Fig. $4 A-F$ ). Malic acid content increased in the cold. At normal light (COLD, COLD+DROUGHT), MvTD10-10 had higher level of malic acid than Mv Makaróni. Additionally, low light under drought (COLD+DROUGHT+LL) caused further increase in this organic acid concentration in Mv Makaróni $(+20 \%)$, contrary to MvTD10-10. Citric acid and isocitric acid contents increased under COLD+DROUGHT+LL as compared to COLD+LL in Mv Makaróni by +32 and $+23 \%$ and in MvTD10-10 by 21 and 38\%, respectively. Cis-aconitic acid accumulation significantly increased by drought in Mv Makaróni $(+25 \%)$ at low light and in MvTD10-10 to a lesser extent (+19\%). Alpha-ketoglutaric acid and succinic acid contents remarkably rose by drought at low light in Mv Makaróni plants (+51 and $+33 \%)$.

The content of sugar alcohol, D-sorbit, equally increased under all COLD conditions in Mv Makaróni. In MvTD10-10, however, it was enhanced under COLD+DROUGHT and COLD+DROUGHT+LL. The total content of D-sorbit was higher in Mv Makaróni than that in MvTD10-10. Additionally, moderate drought treatment increased D-sorbit content by $+91 \%$ at growth light and $+88 \%$ at LL in MvTD10-10 (Fig. 4O). All metabolite data can be found in Table $3 \mathrm{~S}$ (supplement).

The relationship between physiological and metabolic changes under moderate drought and low temperature in durum wheat was illustrated in Fig. 5.

\section{Discussion}

Hardening or acclimation has great importance in plant adaptation. It occurs, when plants are exposed to moderate stress (eustress), thus it can be regarded as a beneficial side effect of adverse environmental factors which enhance fitness (Janda et al. 2014, Savvides et al. 2016). The individual responses of plants to low temperature and drought have been already described in detail, whereas the combined effect of these two stressors on plant assimilation is less well-known (Beck et al. 2007, Pandey et al. 2015). The question arises, how drought affects the cold hardiness in cereal crops, such as durum wheat, with special regard to the photosynthetic light-utilization capability which is a pivotal process of assimilation.

Application of individual drought, heat, and combined drought and heat stress resulted in altered biochemical responses in tomato plants. Combined stress caused a greater drop in relative water content and photosynthetic parameters and changes in other stress indicators as well compared to plants that were exposed to only one certain stress factor. These results suggested that multiple stress caused additional injuries over individual stress in tomato (Raja et al. 2020). In the previous study, neither of the two abiotic factors alleviated the unfavorable effects of the other, and the double stress adversely affected metabolism. Although, drought stress was set to severe and not to a moderate level in the above-mentioned study. High light intensity, especially under abiotic stress, acts synergistically, damages PSII directly or inhibits the repair of PSII after photodamage, and decreases quantum yield (Nishiyama and Murata 2014). In addition, low light under combined stress alleviates photodamage effects.

Previous studies showed that mild water stress protected photosynthetic electron transport components during normal physiological and stress conditions (Cornic and Fresneau 2002, Leverne and Krieger-Liszkay 2021) and the photosynthetic apparatus was highly resistant to moderate drought indicated by unchanged actual quantum yield of PSII contrary to severe drought stress conditions (Cornic and Briantais 1991). In durum wheat, previous results showed that maximal quantum yield, $\mathrm{F}_{\mathrm{v}} / \mathrm{F}_{\mathrm{m}}$, decreased under normal light treatment and it was restored by low light both in irrigated and water-deficient state at low temperature. In contrast to the $F_{v} / F_{m}$, under COLD+DROUGHT+LL, the $\mathrm{Y}_{\text {(II) }}$ remained unchanged as compared to the CONTROL level (Majláth et al. 2016). Analysing the changes of $\mathrm{Chl} a$ fluorescence helped explore that the integrity and operation of photosynthetic machinery strongly depend on the level of water deficit and the ratio of stomatal and nonstomatal limitation in the monocotyledonous perennial ryegrass (Dąbrowski et al. 2019). It suggests the existence of an optimum level of dehydration in plants which may depend on species and support alarm reaction during combined stress conditions. The preliminary findings raised the questions, how the kinetics change during the initial phase of quenching of Chl fluorescence and which are the underlying mechanisms responsible for the protection.

Besides the changes observed in steady-state $\mathrm{Y}_{(\mathrm{II})}$ (Majláth et al. 2016), the present study demonstrated that plants grown under COLD+DROUGHT and COLD+ DROUGHT+LL conditions showed significantly increased 


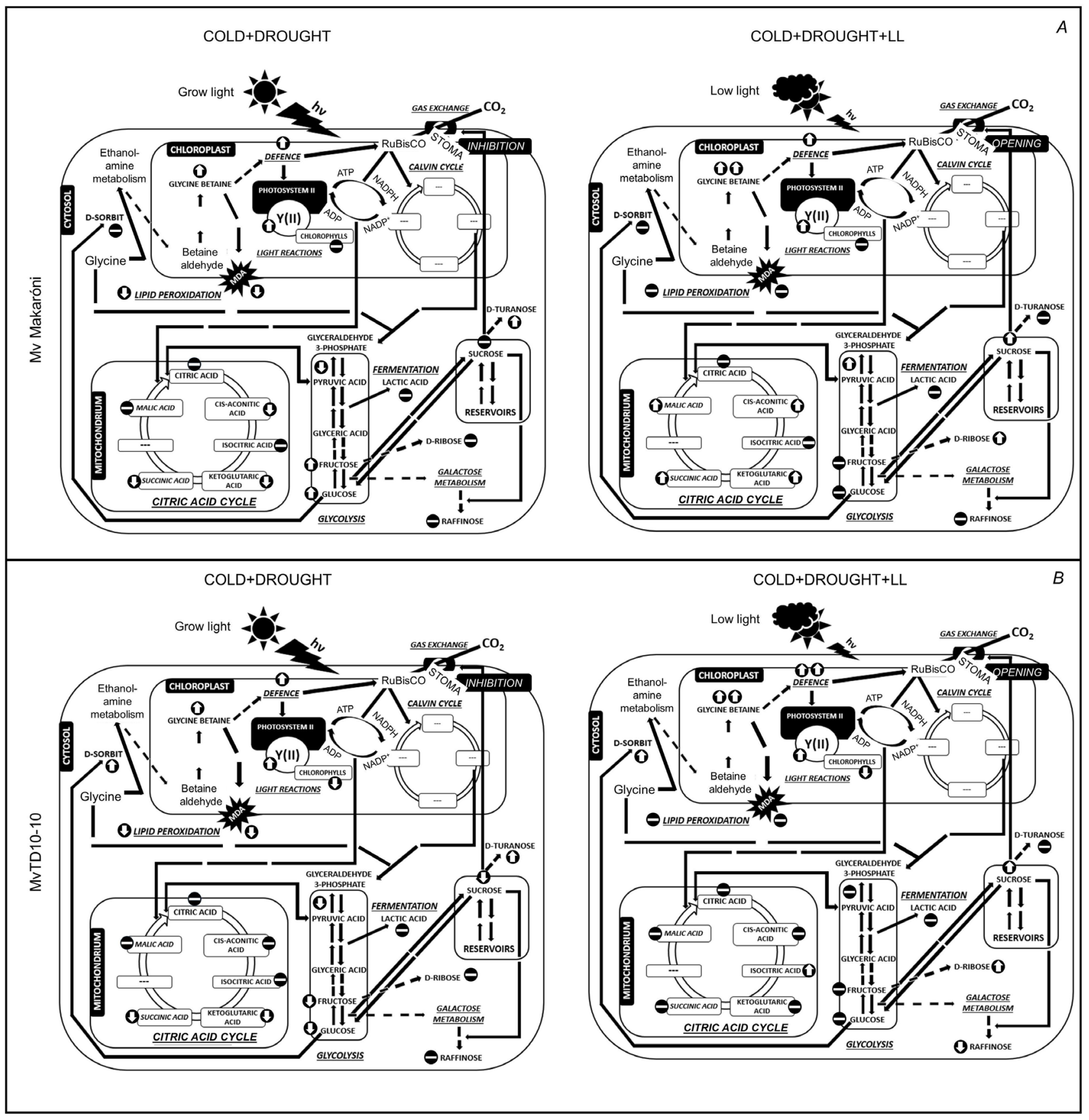

Fig. 5. Schematic overview represents the role of moderate drought acclimation on the photosynthetic and sugar metabolic processes at subotimum temperature in the $(A)$ cold-tolerant Mv Makaróni and $(B)$ cold-sensitive MvTD10-10 durum wheat cultivars. Comparison were made between COLD $v s$. COLD+DROUGHT and COLD+LL $v s$. COLD+DROUGHT+LL and all significant changes $(p \leq 0.05)$ were indicated by upward and downward arrows which indicate activation and inhibition, respectively. Symbol '-' means compounds did not change and '---' means compounds were not investigated.

actual quantum yield. The difference in the formation of steady-state actual quantum yield between the coldtolerant and sensitive durum wheat cultivars was also confirmed. In summary, the present investigation of $\mathrm{Y}_{(\mathrm{II})}$ kinetics provided further data and showed that $\mathrm{Y}_{(\mathrm{II})}$ values were different between the normally irrigated and moderate drought-stressed populations not only at the steady state but during the whole fluorescence quenching period.

The investigation of the values of the initial slope of $Y_{\text {(II) }}$ curves showed that actual PSII quantum yield reached the smallest level at COLD and COLD+LL. The $\mathrm{P}_{\mathrm{d}}$ values suggested that Mv Makaróni responded rapidly to moderate drought and maintained $Y_{(I I)}$ higher at both illumination levels than MVTD10-10. The drought-induced 
elevation in $\mathrm{Y}_{\text {(II) }}$ could be attributed to regulated excitation energy dissipation defence processes $\left[\mathrm{Y}_{(\mathrm{NPQ})}\right]$ rather than to nonregulated processes $\left[\mathrm{Y}_{(\mathrm{NO})}\right]$. These parameters provide information about the fate of the absorbed quanta and the balance between light capture and utilization (Klughammer and Schreiber 2008). In the present study, the kinetics of $\mathrm{Y}_{(\mathrm{NPQ})}$ followed the changes of $\mathrm{Y}_{(\mathrm{II})}$, which indicates that acclimation could be adequate, the regulated ways of excitation energy release were dominant during photoprotection at the expense of nonregulated defence ways. In conclusion, plants grown in the cold were able to utilize the absorbed light more effectively under moderate drought than under irrigated conditions. A recent study reported that moderate water loss in the mesophyll tissue led to the stabilisation of the quinone A and B acceptor components in PSII in spinach plants (Leverne and Krieger-Liszkay 2021). Interestingly, in the case of CONTROL cold-sensitive MvTD10-10 plants, the $\mathrm{Y}_{(\mathrm{NPQ})}$ values remained higher at low light during the initial phase of quenching (2-4.5 $\mathrm{min})$. It may be related to the lowest $\mathrm{Y}_{\text {(II) }}$ observed under COLD+LL and confirms that this cultivar has a greater sensitivity to cold. Thus, MvTD10-10 cold-sensitive cultivar utilized light less effectively under low light. As described earlier, normal light is necessary for the acclimation and the development of cold and frost tolerance at low temperatures (Janda et al. 2014). No differences could be observed in the $\mathrm{Y}_{(\mathrm{NO})}$ kinetics under either moderate drought or irrigated conditions, which also suggests that the protective regulatory mechanisms were adequate.

Changes in $\mathrm{Y}_{(\mathrm{NPQ})}$ reflect either radiative or nonradiative deexcitation pathways. One type of radiative deexcitation is the continuous heat dissipation mediated by Car forms. Whilst Car biosynthesis is under photosynthetic redox control, its contents affect quantum yield parameters (Pizarro and Stange 2009). Reorganization of lightharvesting complexes is another manner of nonradiative deexcitation mechanisms and has a pivotal role to promote the excitation energy transfer to the reaction center chlorophylls (Kramer et al. 2004). In order to understand which mechanisms are responsible for the changes in quantum yield parameters, the photosynthetic pigment composition and MDA content were investigated. According to the data in the literature, drought does not necessarily decrease the $\mathrm{Chl}$ content. Furthermore, a positive correlation was found between the Chl content and quantum yield in durum wheat and barley under drought conditions (Flagella et al. 1994, Li et al. 2006). Car compounds play a role in the radiative deexcitation mechanisms (Choudhury and Behera 2001). The present results showed that the total $\mathrm{Chl}$ and $\mathrm{Car}$ contents decreased with the temperature drop and did not restore by moderate drought. This suggests that the recovery of $\mathrm{Y}_{\text {(II) }}$ induced by drought in the cold cannot be explained by changes in pigment content. It is known that changes in the Chl content do not affect photosynthesis directly, while the integrity of the molecular components of photosystems has a much more important influence on the $\mathrm{Y}_{(\mathrm{II})}$. Membrane decomposition, via peroxidation of polyunsaturated fatty acids, is an accompanying effect of cold, which causes the accumulation of peroxide ions, ROS, and reactive aldehyde forms, such as MDA (Chan et al. 2012, Nejadsadeghi et al. 2015). Light also has a detrimental effect on membrane lipids in the cold (Janda et al. 2014). Degradation of polyunsaturated fatty acids by peroxidation causes alterations in membrane fluidity, which affects the integrity of the photosynthetic machinery and reduces $\mathrm{Y}_{(\mathrm{II})}$. Enhanced lipid peroxidation in Pistacia plants was associated with reduced photoprotection, as indicated by reduced tocopherol contents and nonphotochemical quenching at suboptimal temperature (Juvany et al. 2014). The present results showed that lipid peroxidation might have damaged biological membranes the most under COLD conditions [increased MDA content, higher $\mathrm{Y}_{(\mathrm{NPQ})}$ ], while membrane integrity was less affected under COLD+DROUGHT [decreased MDA content, lower $\left.\mathrm{Y}_{(\mathrm{NPQ})}\right]$. Thus, it seems that changes in MDA and the nonradiative deexcitation mechanisms of $\mathrm{Y}_{(\mathrm{NPQ})}$ may be responsible for the sustenance of $\mathrm{Y}_{\text {(II) }}$ at a suboptimum temperature in durum wheat.

Amongst osmolytes, GB acts as an important defence compound against various stresses, such as cold, excess light, and drought (Ahmad et al. 2013). GB is known to be involved in maintaining the integrity of the photosystems by helping balance the osmotic and redox environment as well as by decreasing lipid peroxidation (Papageorgiou and Murata 1995, Fariduddin et al. 2013). A recent study reported that GB protects chilling-sensitive tomato plants against cold by inducing fatty acid desaturase and lipoxygenase gene expression (Karabudak et al. 2014). Regarding that GB content was generally elevated during low-temperature conditions, except for COLD conditions in this study, enzymes acting against lipid peroxidation processes might be activated which was reflected by the unaltered or decreased MDA content. Additionally, moderate drought raised GB content at both light regimes in the two investigated durum wheat cultivars. The defensive role of GB on photosynthesis under drought was also described in bread wheat (Wang et al. 2010), however, this species does not accumulate significant amounts of GB under natural conditions (Giri 2011). The exogenous application of GB maintained higher $F_{v} / F_{m}$, which recovered more rapidly after photoinhibition (Ma et al. 2006). Our results indicated that $\mathrm{Y}_{\text {(II) }}$ was sustained by regulated water deficiency, which, here, might also be attributed to the GB-induced defence processes. In our study, also greatly elevated GB contents were measured at low temperature under low-light conditions in both cultivars. It is worth mentioning that little knowledge is available on the low-light-dependent accumulation of this osmolyte. Theoretically, as GB has versatile roles in stress defence, its level elevates under stressful circumstances. A similar effect of low light was described on GB accumulation under salinity in durum wheat (Carillo et al. 2011). Glycine is a key member of GB metabolism which is synthesized by a multistep reaction route from pyruvic acid via 3-phosphoglyceric acid, serine, and glycine (Igamberdiev and Kleczkowski 2018). We hypothesize that a decrease in glycolysis intermediates, such as pyruvic acid, under normal growth light and mild- 
drought conditions, may affect glycine formation, and thus, limit GB biosynthesis. Oppositely, pyruvic and maybe 3-phosphoglyceric acid contents were not affected or elevated by low light conditions, which might explain the enhanced, low light-induced accumulation of this osmolyte. In other words, greatly elevated GB contents observed at COLD+DROUGHT+LL may be in a relationship with the turnover rates of glycolytic reactions.

The biosynthesis, remobilization, and conversion of sugars are in an intimate relationship with the outcome of photosynthesis. In this study, all sugars and sugar metabolism-related compounds showed similar contents at CONTROL and DROUGHT stages at $21^{\circ} \mathrm{C}$ (growth temperature). This poses that moderate drought acclimation of wheat plants was efficient and the water shortage applied did not affect plant life adversely. Only sucrose content rose significantly at DROUGHT as compared to CONTROL in Mv Makaróni. Sucrose concentration is considered a sensitive drought-stress marker. Accumulation of sucrose was correlated with increased tolerance for drought and freezing (Mattana et al. 2005). In reaction to low-temperature stress, plants have developed excellent regulatory mechanisms, including changes in gene expression and metabolites. The protein-encoding genes involved in the metabolism of sucrose-starch and the glyoxylate cycle were upregulated and these changes were associated with the accumulation of glucose, fructose, and sucrose in rice after cold or dehydration exposure, as revealed by the study which examined the stressrelated metabolites, phytohormones, and gene transcripts (Maruyama et al. 2014).

Most of the measured carbohydrates and organic acids exhibited similar contents in each treatment combination during the cold phase. Although, there were some exceptions, such as D-sorbit, raffinose, and lactic acid. In addition to the drought-induced accumulation of D-sorbit in Mv Makaróni, the increase of its amount under COLD and COLD+DROUGHT may be in a relationship with the greater cold tolerance of this cultivar. D-sorbit is widely known as a protecting agent against osmotic stress, including low temperature-induced secondary osmotic stress. The higher accumulation of leaf D-sorbit during stress conditions could serve as one of the real components needed for osmotic adjustment (Krasensky and Jonak 2012). To prevent membrane disintegration and enzyme inactivation, plants accumulate certain osmolyte sugars (raffinose and D-sorbit) to decrease the turgor potential along with ROS detoxification by reestablishing the cellular redox level (Mahajan and Tuteja 2005, Krasensky and Jonak 2012). They strengthen the deleterious effects of oxidative stress by protecting enzymes and membranes from stress injury due to the probable antioxidative roles of D-sorbit and proline (Ashraf et al. 2011, de Campos et al. 2011). In addition, D-sorbit accumulation has been associated with drought stress tolerance in most plant species (Krasensky and Jonak 2012). The biochemical responses to drought, primarily D-sorbit and raffinose accumulation, have been consistently linked to toleranceconferred physiological responses to water stress (Jiménez et al. 2013). In conclusion, D-sorbit may have been represented as a possible defence response to cold in the cold-sensitive MvTD10-10 cultivar.

Raffinose is a trisaccharide sugar that is present at high contents under natural and stressful conditions as well and serves as an antioxidant to defend plant cells from oxidative damage and to preserve redox homeostasis (NishizawaYokoi et al. 2008). Raffinose actively accumulates in plants under drought and dehydration (Palma et al. 2014, Pirzadah et al. 2014). These results indicate that raffinose also acts as a free radical scavenger in the chloroplasts of higher plants, which serve as potential sources of antioxidants, along with well-known ROS-scavenging enzymes and nonenzymatic antioxidants (NishizawaYokoi et al. 2008). However, increased raffinose content did not boost freezing tolerance in Arabidopsis plants that constitutively expressed cucumber galactinol synthase (GolS) (Zuther et al. 2004) and neither in gols 1 knock-out plants nor in raffinose synthase knock-out mutants, both of which were impaired in raffinose accumulation, showed any apparent increase in sensitivity to heat or freezing stress (Panikulangara et al. 2004). Raffinose plays a major role in water shortage stress tolerance (Taji et al. 2002). The accumulation of raffinose was observed in vacuoles, cytosol, and chloroplast under cold stress (Gu et al. 2018). In our study, raffinose content also increased by cold, remarkably in the cold-sensitive MvTD10-10 cultivar.

Nonmetabolizable sugars, such as D-turanose, activate different signal transduction pathways of source and sink metabolism relation of sugars and may originate from the plant microflora (Sinha et al. 2002). The presence of D-turanose has been described as a part of the stress-altered metabolome during the fruit development of strawberry (Zhang et al. 2011), in heat-stressed citrus fruits (Yun et al. 2013), in chickpea under high salinity (Dias et al. 2015), and under low-temperature stress of peanut (Wang et al. 2017). However, none of these studies have mentioned the further role of turanose in stress-defence processes. The application of D-turanose has uncovered new sugar-sensing mechanisms (Loreti et al. 2000). In contrast to the metabolizable sugar sucrose, D-turanose rapidly induced the extracellular invertase at mRNA level, whereas the transcript level of ribulose bisphosphate carboxylase was not affected in tomato (Sinha et al. 2002). Overall, D-turanose may have a role against various stresses during plant life, however, the rate of absorption from rhizosphere, transport, and distribution in various organs is of greater importance than its independent biosynthesis in plants.

In our study, D-turanose content was found to be maximal during the physiologically most unfavorable COLD and COLD+DROUGHT conditions. This observation may refer to its emphasized role in sugar signalling. On the other hand, since sucrose content did not change or even slightly decreased under COLD+DROUGHT, glucose catabolism may decelerate and D-turanose content elevated under normal light. In contrast to normal light, low light helped sustain glucose metabolism in a better way than normal light and led to D-turanose accumulation to a 
lesser extent. This finding suggests the role of D-turanose on the defence against combined abiotic stresses in durum wheat.

Moreover, sucrose is an important molecule and can be split into glucose and fructose to provide energy for cellular biosynthesis (Smeekens et al. 2010). It should be also considered that the stomatal opening can be also stimulated by high contents of sucrose (Bates et al. 2012) and facilitated by photosynthesis (Misra et al. 2015).

The increased $\mathrm{O}_{2}$ photoreduction rate in chloroplasts caused oxidative damage, limited $\mathrm{CO}_{2}$ fixation, and higher photorespiration under drought stress (Cruz de Carvalho 2008). Additionally, cold slowed down electron transport rate and may have fortified photorespiration. Stomatal closure rapidly leads to an overreduction of the photosystems in the chloroplast (Nishiyama and Murata 2014, Keech et al. 2017). Under unfavourable conditions, plants produce L-lactate, ethanol, and alanine through fermentation against the overreduction of the $\mathrm{NAD}^{+}-\mathrm{NADH}$ pool. Lactic acid functions as an electron sink (Maurino and Engqvist 2015). In the current study, lactic acid concentration changed in MvTD10-10 only. Drought induced the lactic acid formation at normal light and low light reduced this accumulation. Overall, it can be concluded that lactic acid accumulation may be related to enhanced photorespiration in the cold-sensitive MvTD10-10.

Our results also showed that accumulation of glucose, which is the major product of photosynthesis, was not altered under COLD+DROUGHT. This finding is in agreement with the maintenance of $\mathrm{Y}_{(\mathrm{II})}$ at COLD+ DROUGHT, as compared to the most unfavourable COLD values. Additionally, low light further promoted glucose accumulation which may be in relationship with a lower photoinhibition rate. Regarding sugar storage, the moderate drought did not significantly affect sucrose formation, which further indicates that drought pretreatment was adequate for acclimatization purposes.

Lower accumulation of TCA cycle intermediates at growth light under combined cold and drought may also indicate a higher level of photorespiration and overreduction of $\mathrm{NAD}^{+} / \mathrm{NADH}$ ratio which reduces glycolysis and energy production. Additionally, the fact is that transaconitic acid, a geometric isomer of cis-aconitic acid, is generally more abundant in grasses and the exogenous application of this compound damaged photosynthesis in soybean (Bortolo et al. 2018). The activity of aconitate isomerase did not regulate trans-aconitic acid content but its presence was found to be necessary for the accumulation of trans-aconitic acid in wheat seedlings (Thompson et al. 1997). Taken together, it suggests that the increased content of cis-aconitic acid in the durum wheat genotypes may be due to the inadequate quantity or activity of aconitate isomerase under the multiple abiotic treatment combinations. This finding was also supported by the fact that the amount of cis-isomer was lower in the cold-tolerant Mv Makaróni and MvTD10-10 during CONTROL conditions.

In summary, durum wheat plants grown under mild water deficit and low light intensity exhibited the most optimal photosynthetic performance and sugar turnover rates compared to the other abiotic treatment combinations during cold hardening (Fig. 5). Temporal differences were found in $\mathrm{Y}_{\text {(II) }}$ during the initial, monotonically increasing phase of chlorophyll fluorescence quenching. It corresponded to the level of cold tolerance of cultivars. The cold-tolerant durum cultivar adapted to actinic light more effectively under the acclimation effect of moderate drought independently from light conditions, maintained $\mathrm{Y}_{\text {(II) }}$ higher, and the steady-state was reached more rapidly at low temperature, as compared to the cold-sensitive genotype. These findings confirmed the hypothesized facilitation effect of moderate drought on $\mathrm{Y}_{\text {(II) }}$ in durum wheat. Protective regulatory mechanisms were revealed which may be responsible for the dissipation of excess excitation energy. The molecular machinery of light capture and utilization could be protected by GB in both cultivars. Additionally, D-sorbit molecules may have been responsible for the defence in cold-sensitive genotype. Overall, the integrity of the light phase was supported by a moderate water deficit. Furthermore, water shortage and growth light resulted in low sucrose content, which was a signal for stomatal closure. Thus, decreasing $\mathrm{CO}_{2}$ uptake led to an increased photorespiration and to the deceleration of glucose formation and degradation. In turn, low light and moderate water deficit positively affected sucrose accumulation, which can serve as an additional source to keep glucose concentration higher and maintain glycolysis and energy production at suboptimum temperature.

\section{References}

Ahmad P., Jaleel C.A., Salem M.A. et al.: Roles of enzymatic and nonenzymatic antioxidants in plants during abiotic stress. - Crit. Rev. Biotechnol. 30: 161-175, 2010.

Ahmad R., Lim C.J., Kwon S.Y.: Glycine betaine: a versatile compound with great potential for gene pyramiding to improve crop plant performance against environmental stresses. - Plant Biotechnol. Rep. 7: 49-57, 2013.

Ali Q., Ali S., Iqbal N. et al.: Alpha-tocopherol fertigation confers growth physio-biochemical and qualitative yield enhancement in field grown water deficit wheat (Triticum aestivum L.). - Sci. Rep.-UK 9: 12924, 2019.

Amin B., Mahleghah G., Mahmood H.M.R., Hossein M.: Evaluation of interaction effect of drought stress with ascorbate and salicylic acid on some of physiological and biochemical parameters in okra (Hibiscus esculentus L.). Res. J. Biol. Sci. 4: 380-387, 2009.

Ashraf M., Akram N.A., Al-Qurainy F., Foolad M.R.: Drought tolerance: roles of organic osmolytes, growth regulators, and mineral nutrients. - Adv. Agron. 111: 249-296, 2011.

Bates G.W., Rosenthal D.M., Sun J. et al.: A comparative study of the Arabidopsis thaliana guard-cell transcriptome and its modulation by sucrose. - PLoS ONE 7: e49641, 2012.

Beck E.H., Fettig S., Knake C. et al.: Specific and unspecific responses of plants to cold and drought stress. - J. Biosci. 32: 501-510, 2007.

Bortolo T.D.S.C., Marchiosi R., Viganó J. et al.: Trans-aconitic acid inhibits the growth and photosynthesis of Glycine max.Plant Physiol. Bioch. 132: 490-496, 2018.

Carillo P., Parisi D., Woodrow P. et al.: Salt-induced accumulation of glycine betaine is inhibited by high light in durum wheat. Funct. Plant Biol. 38: 139-150, 2011.

Chan T., Shimizu Y., Pospísil P. et al.: Quality control of photosystem II: Lipid peroxidation accelerates photoinhibition 
under excessive illumination. - PLoS ONE 7: e52100, 2012.

Chemikosova S.B., Pavlencheva N.V., Gur'yanov O.P., Gorshkova T.A.: The effect of soil drought on the phloem fiber development in long-fiber flax. - Russ. J. Plant Physiol. 53: 656-662, 2006.

Choudhury N.K., Behera R.K.: Photoinhibition of photosynthesis: Role of carotenoids in photoprotection of chloroplast constituents. - Photosynthetica 39: 481-488, 2001.

Cornic G., Briantais J.-M.: Partitioning of photosynthetic electron flow between $\mathrm{CO}_{2}$ and $\mathrm{O}_{2}$ reduction in a $\mathrm{C}_{3}$ leaf (Phaseolus vulgaris L.) at different $\mathrm{CO}_{2}$ concentrations and during drought stress. - Planta 183: 178-184, 1991.

Cornic G., Fresneau C.: Photosynthetic carbon reduction and carbon oxidation cycles are the main electron sinks for photosystem II activity during a mild drought. - Ann. Bot.London 89: 887-894, 2002.

Cruz de Carvalho M.H.: Drought stress and reactive oxygen species: production, scavenging and signaling. - Plant Signal. Behav. 3: 156-165, 2008.

Dąbrowski P., Baczewska-Dąbrowska A.H., Kalaji H.M. et al.: Exploration of chlorophyll $a$ fluorescence and plant gas exchange parameters as indicators of drought tolerance in perennial ryegrass. - Sensors-Basel 19: 2736, 2019.

de Campos M.K.F., de Carvalho K., de Souza F.S. et al.: Drought tolerance and antioxidant enzymatic activity in transgenic 'Swingle' citrumelo plants overaccumulating proline. Environ. Exp Bot. 72: 242-250, 2011.

Dias D.A., Hill C.B., Jayasinghe N.S. et al.: Quantitative profiling of polar primary metabolites of two chickpea cultivars with contrasting responses to salinity. - J. Chromatogr. B 1000: $1-13,2015$.

Evers D., Lefèvre I., Legay S. et al.: Identification of droughtresponsive compounds in potato through a combined transcriptomic and targeted metabolite approach. - J. Exp. Bot. 61: 2327-2343, 2010.

Fariduddin Q., Varshney P., Yusuf M. et al.: Dissecting the role of glycine betaine in plants under abiotic stress. - Plant Stress 7: 8-18, 2013.

Fiehn O.: Metabolomics - the link between genotypes and phenotypes. - Plant Mol. Biol. 48: 155-171, 2002.

Flagella Z., Pastore D., Campanile R.G., Di Fonzo N.: Photochemical quenching of chlorophyll fluorescence and drought tolerance in different durum wheat (Triticum durum) cultivars. - J. Agr. Sci. 122: 183-192, 1994.

Giri J.: Glycinebetaine and abiotic stress tolerance in plants. Plant Signal. Behav. 6: 1746-1751, 2011.

Grieve C.M., Grattan S.R.: Rapid assay for determination of water soluble quaternary ammonium compounds. - Plant Soil 70: 303-307, 1983.

Gu H., Lu M., Zhang Z. et al.: Metabolic process of raffinose family oligosaccharides during cold stress and recovery in cucumber leaves. - J. Plant Physiol. 224-225: 112-120, 2018.

Guo R., Shi L., Jiao Y. et al.: Metabolic responses to drought stress in the tissues of drought-tolerant and drought-sensitive wheat genotype seedlings. - AoB Plants 10: ply016, 2018.

Hussain H.A., Hussain S., Khaliq A. et al.: Chilling and drought stresses in crop plants: Implications, cross talk, and potential management opportunities. - Front. Plant Sci. 9: 393, 2018.

Igamberdiev A.U., Kleczkowski L.A.: The glycerate and phosphorylated pathways of serine synthesis in plants: the branches of plant glycolysis linking carbon and nitrogen metabolism. - Front. Plant Sci. 9: 318, 2018.

Janda T., Majláth I., Szalai G.: Interaction of temperature and light in the development of freezing tolerance in plants. J. Plant Growth Regul. 33: 460-469, 2014.

Janda T., Tajti J., Hamow K.Á. et al.: Acclimation of photosynthetic processes and metabolic responses to elevated temperatures in cereals. - Physiol. Plantarum 171: 217-231, 2021.

Jiménez S., Dridi J., Gutiérrez D. et al.: Physiological, biochemical and molecular responses in four Prunus rootstocks submitted to drought stress. - Tree Physiol. 33: 1061-1075, 2013.

Juvany M., Müller M., Pintó-Marijuan M., Munné-Bosch S.: Sexrelated differences in lipid peroxidation and photoprotection in Pistacia lentiscus. - J. Exp. Bot. 65: 1039-1049, 2014.

Kalaji H.M., Jajoo A., Oukarroum A. et al:: Chlorophyll a fluorescence as a tool to monitor physiological status of plants under abiotic stress conditions. - Acta Physiol. Plant. 38: 102, 2016.

Karabudak T., Bor M., Özdemir F., Türkan İ.: Glycine betaine protects tomato (Solanum lycopersicum) plants at low temperature by inducing fatty acid desaturase 7 and lipoxygenase gene expression. - Mol. Biol. Rep. 41: 1401$1410,2014$.

Kaya C., Ashraf M., Wijaya L., Ahmad P.: The putative role of endogenous nitric oxide in brassinosteroid-induced antioxidant defence system in pepper (Capsicum annuиm L.) plants under water stress. - Plant Physiol. Bioch. 143: 119128, 2019.

Kaya C., Şenbayram M., Akram N.A. et al.: Sulfur-enriched leonardite and humic acid soil amendments enhance tolerance to drought and phosphorus deficiency stress in maize (Zea mays L.). - Sci. Rep.-UK 10: 6432, 2020.

Keech O., Gardeström P., Kleczkowski L.A., Rouhier N.: The redox control of photorespiration: from biochemical and physiological aspects to biotechnological considerations. Plant Cell Environ. 40: 553-569, 2017.

Keren N., Krieger-Liszkay A.: Photoinhibition: molecular mechanisms and physiological significance. - Physiol. Plantarum 142: 1-5, 2011.

Klughammer C., Schreiber U.: Saturation Pulse method for assessment of energy conversion in PS I. - PAM Appl. Notes 1: 11-14, 2008.

Kohli S.K., Khanna K., Bhardwaj R. et al.: Assessment of subcellular ROS and NO metabolism in higher plants: multifunctional signaling molecules. - Antioxidants 8: 641, 2019.

Kramer D.M., Avenson T.J., Edwards G.E.: Dynamic flexibility in the light reactions of photosynthesis governed by both electron and proton transfer reactions. - Trends Plant Sci. 9: 349-357, 2004.

Krasensky J., Jonak C.: Drought, salt, and temperature stressinduced metabolic rearrangements and regulatory networks. J. Exp. Bot. 63: 1593-1608, 2012.

Leverne L., Krieger-Liszkay A.: Moderate drought stress stabilizes the primary quinone acceptor $\mathrm{Q}_{\mathrm{A}}$ and the secondary quinone acceptor $\mathrm{Q}_{\mathrm{B}}$ in photosystem II. - Physiol. Plantarum 171: 260-267, 2021.

Li R.H., Guo P.P., Baum M. et al.: Evaluation of chlorophyll content and fluorescence parameters as indicators of drought tolerance in barley. - Agr. Sci. China 5: 751-757, 2006.

Lichtenthaler H.K.: Chlorophylls and carotenoids: pigments of photosynthetic biomembranes. - Method. Enzymol. 148: 350382, 1987.

Loreti E., Amedo A., Perata P.: Glucose and disaccharide mechanisms modulate the expression of $\alpha$-amylase in barley embryos. - Plant Physiol. 123: 939-948, 2000.

Ma Q.Q., Wang W., Li Y.H. et al.: Alleviation of photoinhibition in drought-stressed wheat (Triticum aestivum) by foliarapplied glycinebetaine. - J. Plant Physiol. 163: 165-175, 2006.

Mahajan S., Tuteja N.: Cold, salinity and drought stresses: An overview. - Arch. Biochem. Biophys. 444: 139-158, 2005.

Majláth I., Darko E., Palla B. et al.: Reduced light and moderate water deficiency sustain nitrogen assimilation and sucrose 
degradation at low temperature in durum wheat. - J. Plant Physiol. 191: 149-158, 2016.

Mäkelä P., Kärkkäinen J., Somersalo S.: Effect of glycinebetaine on chloroplast ultrastructure, chlorophyll and protein content, and RuBPCO activities in tomato grown under drought or salinity. - Biol. Plantarum 43: 471-475, 2000.

Maruyama K., Urano K., Yoshiwara K. et al.: Integrated analysis of the effects of cold and dehydration on rice metabolites, phytohormones, and gene transcripts. - Plant Physiol. 164: 1759-1771, 2014.

Mattana M., Biazzi E., Consonni R. et al.: Overexpression of osmyb4 enhances compatible solute accumulation and increases stress tolerance of Arabidopsis thaliana. - Physiol. Plantarum 125: 212-223, 2005.

Maurino V.G., Engqvist M.K.: 2-hydroxy acids in plant metabolism. - In: The Arabidopsis Book. Vol. 13. Pp. e0182. The American Society of Plant Biologists, 2015.

Misra B.B., Acharya B.R., Granot D. et al.: The guard cell metabolome: functions in stomatal movement and global food security. - Front. Plant Sci. 6: 334, 2015.

Mittler R.: Abiotic stress, the field environment and stress combination. - Trends Plant Sci. 11: 15-19, 2006.

Miura K., Furumoto T.: Cold signaling and cold response in plants. - Int. J. Mol. Sci. 14: 5312-5337, 2013.

Nath K., O'Donnell J.P., Lu Y.: Chlorophyll fluorescence for high-throughput screening of plants during abiotic stress, aging, and genetic perturbation. - In: Hou H., Najafpour M., Moore G., Allakhverdiev S. (ed.): Photosynthesis: Structures, Mechanisms, and Applications. Pp. 261-273. Springer, Cham 2017.

Nejadsadeghi L., Maali-Amiri R., Zeinali H. et al.: Membrane fatty acid compositions and cold-induced response in tetraploid and hexaploid wheats. - Mol. Biol. Rep. 42: 363372, 2015.

Nishiyama Y., Murata N.: Revised scheme for the mechanism of photoinhibition and its application to enhance the abiotic stress tolerance of the photosynthetic machinery. - Appl. Microbiol. Biot. 98: 8777-8796, 2014.

Nishizawa-Yokoi A., Yabuta Y., Shigeoka S.: The contribution of carbohydrates including raffinose family oligosaccharides and sugar alcohols to protection of plant cells from oxidative damage. - Plant Signal. Behav. 3: 1016-1018, 2008.

Palma F., Carvajal F., Lluch C. et al.: Changes in carbohydrate content in zucchini fruit (Cucurbita pepo L.) under low temperature stress. - Plant Sci. 217-218: 78-86, 2014.

Pandey P., Irulappan V., Bagavathiannan M.V., Senthil-Kumar M.: Impact of combined abiotic and biotic stresses on plant growth and avenues for crop improvement by exploiting physio-morphological traits. - Front. Plant Sci. 8: 537, 2017.

Pandey P., Ramegowda V., Senthil-Kumar M.: Shared and unique responses of plants to multiple individual stresses and stress combinations: physiological and molecular mechanisms. Front. Plant Sci. 6: 723, 2015.

Panikulangara T.J., Eggers-Schumacher G., Wunderlich M. et al.: Galactinol synthase1. A novel heat shock factor target gene responsible for heat-induced synthesis of raffinose family oligosaccharides in Arabidopsis. - Plant Physiol. 136: 31483158, 2004.

Papageorgiou G.C., Murata N.: The unusually strong stabilizing effects of glycinebetaine on the structure and function of the oxygen-evolving photosystem II complex. - Photosynth. Res. 44: 243-252, 1995.

Pirzadah B.T., Malik B., Rehman U.R. et al.: Signaling in response to cold stress. - In: Hakeem R.K., Rehman U.R., Tahir I. (ed.): Plant Signaling: Understanding the Molecular
Crosstalk. Pp. 193-226. Springer, New Delhi 2014.

Pizarro L., Stange C.: Light-dependent regulation of carotenoid biosynthesis in plants. - Cienc. Inv. Agr. 36: 143-162, 2009.

Raja V., Qadir S.U., Alyemeni M.N., Ahmad P.: Impact of drought and heat stress individually and in combination on physio-biochemical parameters, antioxidant responses, and gene expression in Solanum lycopersicum. -3 Biotech 10: 208, 2020.

Ruelland E., Vaultier M.-N., Zachowski A., Hurry V.: Cold signalling and cold acclimation in plants. - Adv. Bot. Res. 49: 36-150, 2009.

Savvides A., Shawkat A., Tester M., Fotopoulos V.: Chemical priming of plants against multiple abiotic stresses: Mission possible? - Trends Plant Sci. 21: 329-340, 2016.

Saxena S.C., Kaur H., Verma P. et al.: Osmoprotectants: potential for crop improvement under adverse conditions. - In: Tuteja N., Singh Gill S. (ed.): Plant Acclimation to Environmental Stress. Pp. 197-232. Springer, New York 2013.

Sharma P., Jha A.B., Dubey R.S., Pessarakli M.: Reactive oxygen species, oxidative damage, and antioxidative defense mechanism in plants under stressful conditions. - J. Bot. 2012: 217037, 2012.

Sinha A.K., Hofmann M.G., Römer U. et al.: Metabolizable and non-metabolizable sugars activate different signal transduction pathways in tomato. - Plant Physiol. 128: 1480-1489, 2002.

Smeekens S., Ma J.K., Hanson J., Rolland F.: Sugar signals and molecular networks controlling plant growth. - Curr. Opin. Plant Biol. 13: 273-278, 2010.

Sohag A.A.M., Tahjib-Ul-Arif M., Brestic M. et al.: Exogenous salicylic acid and hydrogen peroxide attenuate drought stress in rice. - Plant Soil Environ. 66: 7-13, 2020.

Taji T., Ohsumi C., Iuchi S. et al.: Important roles of drought- and cold-inducible genes for galactinol synthase in stress tolerance in Arabidopsis thaliana. - Plant J. 29: 417-426, 2002.

Takahashi S., Murata N.: How do environmental stresses accelerate photoinhibition? - Trends Plant Sci. 13: 178-182, 2008.

Thomas J.C., Perron M., Davies E.C.: Genetic responsiveness to copper in the ice plant, Mesembryanthemum crystallinum. Plant Sci. 167: 259-266, 2004.

Thompson J.F., Schaefer S.C., Madison J.T.: Role of aconitate isomerase in trans-aconitate accumulation in plants. - J. Agr. Food Chem. 45: 3684-3688, 1997.

Wang G.P., Zhang X.Y., Li F. et al.: Overaccumulation of glycine betaine enhances tolerance to drought and heat stress in wheat leaves in the protection of photosynthesis. - Photosynthetica 48: 117-126, 2010.

Wang X.J., Sun D.L., Bian N.F. et al.: Metabolic changes of peanut (Arachis hypogaea L.) buds in response to low temperature (LT). - S. Afr. J. Bot. 111: 341-345, 2017.

Yun Z., Gao H., Liu P. et al:: Comparative proteomic and metabolomic profiling of citrus fruit with enhancement of disease resistance by postharvest heat treatment. - BMC Plant Biol. 13: 44, 2013.

Zhang J., Wang X., Yu O. et al.: Metabolic profiling of strawberry (Fragaria $\times$ ananassa Duch.) during fruit development and maturation. - J. Exp. Bot. 62: 1103-1118, 2011.

Zhang J., Yang D., Li M., Shi L.: Metabolic profiles reveal changes in wild and cultivated soybean seedling leaves under salt stress. - PLoS ONE 11: e0159622, 2016.

Zuther E., Büchel K., Hundertmark M. et al.: The role of raffinose in the cold acclimation of Arabidopsis thaliana. - FEBS Lett. 576: 169-173, 2004.

(C) The authors. This is an open access article distributed under the terms of the Creative Commons BY-NC-ND Licence. 